\title{
ON $K$-SEMIMETRIC SPACES
}

\author{
DENNIS K. BURKE
}

\begin{abstract}
An example is constructed of a separable Moore space that does not possess a compatible $K$-semimetric.
\end{abstract}

A semimetric $d$ for a space $X$ is said to be a $K$-semimetric if $d(H, K)>0$ whenever $H$ and $K$ are disjoint compact subsets of $X$. It is the purpose of this note to provide an example of a regular semimetrizable space (in fact, a separable Moore space) which does not have a compatible $K$-semimetric. This answers a question first posed by A. V. Arhangel'skii in [1] and later by others such as H. Martin in [3].

The description of the example follows below. The sets $R, P, Q$, and $N$ denote the real numbers, irrational numbers, rational numbers, and natural numbers respectively.

EXAMPLE 1. A separable Moore space which is not $K$-semimetrizable.

Let $A_{1}=P \times\{0\}, A_{2}=P \times\{-1\}, E=\{(r, s) \in Q \times Q: s>0\}$, and $X=A_{1} \cup A_{2} \cup E$. Describe a local base for points in $X$ as follows: Points in $E$ have the usual neighborhoods (as inherited from $R \times R$ ). If $a \in P$ and $n \in N$ let

$$
\begin{aligned}
U_{n}(a, 0) & =\{(a, 0)\} \cup\{(r, s) \in E: a<r<s / n+a, s<1 / n\}, \\
U_{n}(a,-1) & =\{(a,-1)\} \cup\{(r, s) \in E:-s / n+a<r<a, s<1 / n\} .
\end{aligned}
$$

Then $\left\{U_{n}(a, 0)\right\}_{1}^{\infty}$, and $\left\{U_{n}(a,-1)\right\}_{1}^{\infty}$, give local bases at $(a, 0) \in A_{1}$ and $(a,-1) \in A_{2}$ respectively. (A simple sketch reveals that $U_{n}(a, 0)$ is $(a, 0)$ along with the "right half of the interior of a $V$ neighborhood at $(a, 0)$ " and $U_{n}(a,-1)$ is $(a,-1)$ along with the "left half".) It is easily verified that $X$ (with the new topology) is a separable, completely regular Moore space.

Let $d$ be a semimetric for $X$-we show that $d$ is not a $K$-semimetric.

For $n \in N$ let

$$
P(n)=\left\{a \in P: d(x, y) \geqslant 1 / n, \text { all } x \in U_{n}(a, 0), y \in U_{n}(a,-1)\right\} .
$$

If $P=\cup_{n=1}^{\infty} P(n)$ there is some $k \in N$ such that $T=\operatorname{int}_{R}\left(\mathrm{cl}_{R}, P(k)\right) \neq \varnothing$. It is possible to find $\left(t_{1}, s\right),\left(t_{2}, s\right) \in E \cap(T \times R)$ and $b \in P(k)$ such that

$$
d\left(\left(t_{1}, s\right),\left(t_{2}, s\right)\right)<\frac{1}{k}, \quad\left|t_{1}-t_{2}\right|<\frac{1}{k(k+1)}, \quad s=\frac{1}{k+1}
$$

Received by the editors November 21, 1977 and, in revised form, January 12, 1978.

AMS (MOS) subject classifications (1970). Primary 54E25; Secondary 54E30.

$K e y$ words and phrases. Semimetric, $K$-semimetric, Moore space, submetrizable, quasimetric regular $G_{\delta}$-diagonal. 
and $t_{1}<b<t_{2}$. If $x=\left(t_{2}, s\right), y=\left(t_{1}, s\right)$ then $x \in U_{k}(b, 0), y \in U_{k}(b,-1)$ and $d(x, y)<1 / k$ which contradicts the definition of $P(k)$. It follows that $\cup_{n=1}^{\infty} P(n) \neq P$ and there exists some

$$
a \in P-\left(\bigcup_{n=1}^{\infty} P(n)\right)
$$

Now $a \notin P(n)$ implies there exists $x_{n} \in U_{n}(a, 0)$ and $y_{n} \in U_{n}(a,-1)$ such that $d\left(x_{n}, y_{n}\right)<1 / n$. Clearly $x_{n} \rightarrow(a, 0)$ and $y_{n} \rightarrow(a,-1)$. If $H=\left\{x_{n}\right\}_{1}^{\infty} \cup$ $\{(a, 0)\}$ and $K=\left\{y_{n}\right\}_{1}^{\infty} \cup\{(a,-1)\}$ then $K$ and $H$ are disjoint compact subsets of $X$, but $d(H, K)=0$; thus $d$ is not a $K$-semimetric for $X$ and the proof is complete.

REMARKS 2. (a) It is trivial that every metric space is $K$-semimetrizable. In fact, A. V. Arhangel'skii [1] has shown that a regular space $Y$ is metrizable if and only if there is a compatible semimetric $d$ for $Y$ such that $d(A, B)>0$ whenever $A$ and $B$ are disjoint subsets of $Y$ with $A$ compact and $B$ closed.

(b) It is known [1] that a semimetric space which is submetrizable (has a weaker metric topology) is also $K$-semimetrizable. W. Lindgren has pointed out to the author that a semimetric space with a coarser $T_{2}$ quasimetric topology is $K$-semimetrizable and that apparently, Example 1 gives the first known example of a Moore space that does not admit a coarser $T_{2}$ quasimetric topology.

(c) Let $X$ be the space of Example 1 and let $Y$ be the quotient space obtained from $X$ by identifying points $(a, 0)$ and $(a,-1)$ for each $a \in P$. Then $Y$ is a completely regular separable submetrizable Moore space. If $f$ : $X \rightarrow Y$ is the corresponding quotient mapping then $f$ is a perfect map from the nonsubmetrizable space $X$ onto a submetrizable Moore space $Y$. This should be contrasted with the result by Borges [2] and Okuyama [4] that if $g$ : $Z \rightarrow M$ is a perfect map from a Hausdorff space $Z$ onto a metric space $M$ then $Z$ is metrizable if and only if $Z$ has a $G_{\delta}$-diagonal. This suggests the following question.

Question 1. If $g: Z \rightarrow M$ is a perfect map from a regular space $Z$ onto a submetrizable space $M$ what minimal diagonal condition on $Z$ will ensure that $Z$ is submetrizable? G. M. Reed has an example [6, Example 3] that shows $Z$ need not be submetrizable even if $Z$ has a regular $G_{\delta}$-diagonal.

Besides the submetrizable condition mentioned in Remark 2(b) different authors have given various sufficient conditions for a Moore space to be $K$-semimetrizable. H. Martin showed that a locally connected rim compact space is $K$-semimetrizable if and only if it is a developable $\gamma$-space [3]. A result by $\mathrm{P}$. Zenor shows that a Moore space with a regular $G_{\delta}$-diagonal is $K$-semimetrizable [5].

QUESTION 2. What minimal topological condition on a Moore space (or semimetric space) will ensure that the space be $K$-semimetrizable? For 
example, is every locally connected rim compact Moore space necessarily $K$-semimetrizable?

\section{REFERENCES}

1. A. V. Arhangel'skii, Mappings and spaces, Uspehi Mat. Nauk 21 (1966), no. 4 (130), 133-184 = Russian Math. Surveys 21 (1966), no. 4, 115-162. MR 37 \#3534.

2. C. R. Borges, On stratifiable spaces, Pacific J. Math. 17 (1966), 1-16.

3. H. W. Martin, Local connectedness in developable spaces, Pacific J. Math. 61 (1975), 219-224.

4. A. Okuyama, On metrizability of M-spaces, Proc. Japan Acad. 40 (1964), 176-179.

5. P. L. Zenor, On spaces with regular $G_{\delta}$-diagonals, Pacific J. Math. 40 (1972), 759-763.

6. G. M. Reed, Some Moore examples, Notices Amer. Math. Soc. 22 (1975), Abstract 726-54-3, p. A-582.

Department of Mathematics, Miami University, OXford, OHo 45056 